\title{
Energy Dissipation in Cremical Reactions on Ultrafast Timescales
}

\author{
D.J. Russell, M.E. Paige, and C.B. Harris
}

\author{
Departrent of Chemistry \\ Univer sity of California \\ and \\ Chemical Sciences Division \\ Lawrence Berkeley Laboratory \\ University of California \\ Berkeley, CA 94720
}

\section{DISCLAIMER}

\begin{abstract}
This report was prepared as an account of work sponsored by an agency of the United States Government. Neither the United States Government nor any asency thereof, nor any of their emptoyecs, makes any warranty, express or implied, or assumes any tegal liability or responsibility for the accuracy, comptetenes, or usefulaest of any information, apparatus, product, or process disclooed, or tepresents that its use would not infringe privately owned rights. Reference herein to any specific commercial product, process, or service by trade name, trademark, manufacturer, or otherwive does not necessarily constitute or imply its endorement, recommeadation, or favoring by the United States Government or any agency thereof. The views and opinions of autbors expresued herein do nol necessarily state or reflect thoue of the United Stales Goverament or any agency thereof.
\end{abstract}

This work was supported in par by a grant by the National Science Foundation and in part by the Office of Naval Research, and by the Director, Office of Energy Research, Oftice of Basic Energy Sciences, Chemical Sciences Division, of the U.S. Deparument of Energy under Contract No. DE-AC03-76SF00098. 
ENERGY DISSIPATION IN CHEMICAL REACTIONS ON ULTRAFAST TIMESCALES

Daniel J. Russell, Mark E. Paige, and Charles B. Harris

Department of Chemistry

University of california

Berkeley, CA 94720

U. S. A.

Abstract

A series of picosecond experiments and computer imulations will be presented that test collistonal and hydrodynamic models for vibrational relaxation in liquids. The relationships between isolated binary collision models (IBC) and stochastic dyamics will be presented. The appropriateness of IBC theory in de scribing vibrational relaxation in liquids will also be discussed. 
A proper description of vibrational energy transfer is essential to the development of chemical reaction theory. In liquids, progress in this area is far behind that which has been made in other phases because of the complexity inherent to this phase. Generally, two approaches have been taken in attempting to model the vibrational relaxation of an excited oscillator in solution. One approach takes the perspective of the excited molecul being solvated in a continuous viscoelastic media which exerts a frictional force on the molecule. The most general form of this interaction is described by the generalized Langevin equation:

$$
\operatorname{ma}(t)=-\int_{t_{0}}^{t} d t^{\prime} m \gamma\left(t^{\prime}\right) v\left(t-t^{\prime}\right)+R(t)
$$

where $m$ is the mass of the particle, $R(t)$ is the random force, and $\gamma(t)$ is the memory function which provides the friction. In this description, the noninstantaneous response of the media to the motions of the oscillator are included. The difficulty with this method has been in developing a model of determining the form of $\gamma(t)$ [the frequency dependence of the friction]. Very early during the theoretical development of vibrational relaxation in liquids, prior to use of the generalized Langevin model, a simpler form of this model known as the Langevin equation was used to describe vibrational relaxation in solution.' According to the Lancjevin equation,

$$
\operatorname{ma}(t)=-\gamma v(t)+R(t)
$$


In the Langevin model, $y$, the friction coefficient, is a constant and thus, the solvent is assumed to respond instantaneously to all oscillator motions. While this model has been successful in describing some phenomenon where the solute moves slowly in relation to the solvent molecules, it has failed in the modeling of vibrational relaxation where the oscillator motions can be quite rast in comparison to that of the solvent. ${ }^{2}$

An alternative perspective adapted to describing vibrational relaxation in solution is that based on the molecular nature of the solvent-solute interactions. In this framework, the interactions which are important to vibrational relaxation are assumed to be dominated by isolated binary collisions between the solvent molecules and the oscillator, fust as they occur in the gas phase. This model known as the Isolated Blnary Collision Model (IBC) states that $K_{i-j}(p, T)$, the relaxation rate of vibrational level $i$ to $j$, is:

$$
K_{1-j}(p, T)=P_{1-j} \cdot Z(p, T)
$$

where $P_{i-j}(T)$ is the probability of $i-j$ per collision, $z(p, T)$ is the oscillator-solvent collision frequency, $p$ is the solvent density, and $T$ is the solvent temperature. Since $P_{i-j}$ is independent of density, the difference between relaxation in the liquid and gas phases at constant $\mathrm{T}$ is solely given by $\mathrm{Z}(\rho)$. Thus, multibody effects (i.e., correlations between collisions) are considered to be inconsequential in this model. The validity of this assumption has been the subject of a longstanding debate in the literature.

As a test system for IBC theory, we have examined its applicability to experimental measurements and computer simulations 
of the ground state vibrational relaxation of geminately recombined $I_{2}$ in liquid $x e$. Following photodissoclation and recombination on the ground state surface, the $I_{2}$ has an excess of $12500 \mathrm{~cm}^{-1}$ of vibrational energy which it then dissipates to the solvent. This relaxation process occurs over $100 \mathrm{I}_{2}$ vibrational levels with the vibrational spacing varying from 0 to $214 \mathrm{~cm}^{-1}$. Since $I_{2}$ contains only one vibrational degree of freedom, the role of the solvent in dissipating the excess vibrational energy of the excited $I_{2}$ can be studied with this system without competition from intramolecular vibrational energy transfer. Furthermore, Xe is an ideal choice as the solvent because of its spherical geometry which makes computations on this system easier and eliminates all but the translational degrees of freedom as the solvent energy accepting modes.

IBC theory is not expected to be valid for this system for two reasons. First, the low vibrational frequencles involved are comparable to the expected collision frequencies. Thus, interference between collisions would be expected to occur. second, IBC theory should not be applicable to a highly excited oscillator in solution as the collisions will be driven by the large amplitude motions of the oscillator rather than occurring at random intervals resulting from the solvent motion. In contrast, previous studies of the IBC theory's validity at liquid density have focussed on the density dependence of the $v=1 \rightarrow 0$ time of high frequency oscillators such as $H_{2}$ and $N_{2}$ where the above conditions to not exist and IBC theory may be valid. ${ }^{3}$

Additionally, study of the multilevel relaxation process of $I_{2}$ 
offers a unique perspective on the validity of the IBC model as compared to these other experiments. The difficulty with interpreting the results of the $v=1 \rightarrow 0$ studies is that a calculation of $\mathrm{z}(p)$ is necessary in order to ascertain the validity of the IBC model since the relative change in relaxation rates between two densities $p_{1}$ and $p_{2}$ according to the IBC model is given by:

$$
\frac{K_{1-f}\left(p_{2}\right)}{K_{1-f}\left(p_{2}\right)}=\frac{Z\left(p_{1}\right)}{Z\left(p_{2}\right)}
$$

In solution, a coliision is a nebulous concept and calculations of 2 vary widely in magnitude with model and are very sensitive to parameters such as molecular size. Thus, studies in which the relaxation rate between two levels is measured as a function of density do not test the underlying assumption of the density independence of $P_{i-j}$ separately from the modei of $z(p)$ employed. In the experiments on $I_{2}$, the relaxation has been experimentally measured over approximately 30 vibrational levels (we could not directly observe the relaxation over the upper half of the ground state surface). Following the relaxation over a large number of vibrational levels as a function of solvent density eliminates the need of calculating $z$ in testing the density dependence of the $P_{i \rightarrow j}$. To understand this distinction, consider the relaxation process over many vibrational levels in the IBC framework. After $z(\rho) \cdot t$ binary collisions, the vibrational population distribution vector, $N(t)$, would be

$$
N(t)=P \circ P \circ P \ldots \cdot P * N(t=0)=P * t \cdot N(t=0)
$$

where $P$ is the matrix of relaxation probabilities per collision for 
transitions between all levels of the syaten. The role of $z(p)$ in this description of the relaxation is to control the timescale of the relaxation process. If the time were normalized by $z(p), 1 . e .$, $t^{\prime}=t / 2(\rho)$, then the resulting $N\left(t^{\prime}\right)$ would be ldentical for all $\rho$. Hence, plots of the average energy of the system as a function of time for different density should show the same functional form, differing only by a linear scaling of the time axis which accounts for the change in $\mathrm{Z}(\rho)$. Provided $\mathrm{Z}(\rho)$ does not vary with vibrational energy, any deviation from linear scaling would be an indication of nonbinary behavior. The timescaling factor $S$ necessary to overlap the energy decay curve at $p_{2}$ to that of $p_{1}$ can be determined from

$$
Z\left(p_{1}\right) \cdot t=s \cdot Z\left(p_{2}\right) \cdot t
$$

which leads to

$$
s=\frac{z\left(p_{1}\right)}{Z\left(p_{2}\right)}
$$

The $I_{2}$ vibrational energy as a function of time is determined experimentally through picosecond transient absorption spectroscopy. Due to the change of the Franck-condon factors with vibrational energy, the transient absorption shifts from the near infrared at high vibrational energies to approximately $520 \mathrm{~nm}$ at $v=0$. With the transient absorptions in this wavelength region and by calculating the extinction coefficients for the ground state absorption, the vibrational population distribution can be determined as a function of time by applying Beer's law. This analysis has been performed for the lower $6000 \mathrm{~cm}^{-1}$ of the $I_{2}$ ground 
state potential surface. The density range of $x_{0}$ in which the experiment was performed was 1.8 to $3.4 \mathrm{~g} / \mathrm{Cc}$ at $280 \mathrm{~K}$ which corresponds to a Lennard-Jones reduced density $\left(\rho \sigma^{3}\right)$ range of 0.57 to 1.07 at a Lennard-Jones reduced temperature (kT/6) of 1.26 . A sample plot of vibrational energy vs. time for the $1.8 \mathrm{~g} / \mathrm{cc}$ and the time scaled $3.0 \mathrm{~g} / \mathrm{cc}$ is shown in the figure 1 . The functional form of the relaxation at all densities is the same. Thus, the $P_{i-1}$ are density independent over the entire liquid density portion of the Xe phase diagram as is assumed in the IEC model.

Concurrent with the exgeriments, a theoretical model of the $I_{2} /$ Xe system was constructed. In order to provide a reference system for comparison of IBC and continuum theories, a molecular dynamics simulation of $I_{2} / X \bullet$ was performed. Comparisons of the predictions of these theories with the simulation results will therefore not depend upon diflerences in the potentials assumed (in contrast to comparisons with the experimental results)." The system was a classical molecular dynamics simulation using periodic boundary conditions. The potentials used were a Lennard-Jones potential between the iodine atoms and Xe atoms and a RKR potential for $I_{2}$. There were 255 xenon atoms and one iodine molecule in the system, these numbers were chosen because they minimized the effects of heating of the liquid after dissociation due to the small number of particles and was it still a small enough system to allow a reasonable number of tiajectories to calculated on a cray $x$-MP. The functional forms of the vibrational energy vs. time for all the densities performed were the same. This would seem to indicate as described above that the $P_{i \rightarrow j}$ are density independent. 
Also, the functional form found from the molecular dynamics simulations was seemingly the same functional form found in the experiments. The only difference was that the molecular dynamics relaxed about 12.4 times faster than the experiment. This may be due in part to the finite volume of xe heating up from the dissociation energy of the lodine. Also, the Lennard-Jones potential may be steeper than the real $I_{2}-x e$ potential. Qualitatively, the density dependence of the relaxation was the same for both the molecular dynamics and the experiment. There was some discrepancy in the actual numbers. Over the liquid range studied by the experiment, from $1.8 \mathrm{~g} / \mathrm{cc}$ to $3.4 \mathrm{~g} / \mathrm{cc}$, the vibrational relaxation was 4 times faster. The molecular dynamics was also 4 times faster for the range of $1.8 \mathrm{~g} / \mathrm{cc}$ to $3.0 \mathrm{~g} / \mathrm{cc}$. The molecular dynamics could not be run at $3.4 \mathrm{~g} / \mathrm{cc}$ because that is a Lennard-Jones solid while real $\mathrm{Xe}$ is a lluid. This shows that the molecular dynamics seems to be a fairly realistic representation of the vibrational relaxation in the liquid and only fails mainly to lack of exact potentials.

Given the success of reproducing experimental results by the molecular dynamics and the evidence that IBC theory may provide a valid model for explaining the experimental results, an IBC calculation was performed. The calculation of $P_{i-j}$ performed for $I_{2}$ and $X e$ is a one dimensional classical calculation of energy loss. Studies have shown that the one dimensional calculation is a reasonable substitute for three dimensions if the constraints described in a paper by McKenzie are realized. ${ }^{5}$ The I-I potential used is the same RKR surface as used in the molecular dynamics 
simulations. The potential between $I$ and $x e$ is a Weeks chandler Anderson (WCA) decomposition of the Lennard-Jones potential. 6 Note that the WCA decomposition wes originally intended to explain liquid structures for reduced densities greater that 0.6 . Even though most of the comparisons to molecular dynamics will be in this range, WCa was not chosen for this reason.

$$
\begin{gathered}
V(r)=4 \varepsilon\left[\left(\frac{\sigma}{r}\right)^{12}-\left[\left(\frac{\sigma}{r}\right)^{c}\right]+c \text { where } r<r_{c}\right. \\
C=4 e\left[\left(\frac{\sigma}{I_{c}}\right)^{12}-\left(\frac{\sigma}{I_{c}}\right)^{\sigma}\right] \text { where } r_{c}=2^{\left(\frac{1}{6}\right)} \sigma \\
V(r)=0 \text {. where } r>r_{c}
\end{gathered}
$$

The WCA decomposition was chosen for three reasons. When a gas liquifies, energy (the heat of vaporization) is released due to the solvent atoms spending most of their time in the bottom of the well where the potential is repulsive. The attractive part of the potential is defined as the part of the potential where the accelerations are negative. Note that in the Lennard-Jones potential, the potential energy may bo negative for $r<02^{1 / 6}$, but the accelerations are not negative. Since the liquid rarely samples the attractive part of the potential, it "as thought that the IBC simulation would be more realistic if it also did not sample that part of the potential. The turning point in the gas phase will also be on average at a smaller radius than in the liquid due to the heat of vaporization, however, the one dimensional model's turning point should be comparable to the molecular dynamics simulation due to the use of the wCA potential. Secondly, not having an attractive section of the potential makes the integration of the trajectory much quicker since there is less 
distance to integrate over and there is no possibility of forming a long lived complex. Thirdly, the molecular dynamics simulation that the one dimensional trajectories would be compared to used a Lennard-Jones potential and the wCA decomposition is the closest approximation to the Lennard-Jones potential within the above constraints.

The trajectories show qualitatively what you would expect keeping in mind $I_{2}{ }^{\prime} s$ anharmonicity. Vibrational energy transfer increases non linearly as a function of $v$, the vibrational quantum number. This at variance to Landau Teller theory, which predicts a linear increase with $v$. Of course, Landau Teller theory is based on a harmonic oscillator and $I_{2}$ is most derinitely nct a harmonic oscillator. A calculation of $I_{2}$ 's average vibrational energy vs. time was performed using data provided by the one dimensional calculations and it was found that the functional form of the energy loss was the same as both the molecular dynamics simulations and the experiments.

In order for IBC theory to be a useful theory it must also be able to make quantitative predictions of the relaxation for a particular density and predictions of the density dependence. The collision rate of 4.5 per ps., which was required to fit the molecular dynamics simulation, is a quite reasonable first order guess of what the collision rate should be for xe at $1.8 \mathrm{~g} / \mathrm{cc}$. since the trajectory calculations were one dimensional, there must be some steric weighting factor to take into account that some collisions are not collinear. It is not clear what that factor should be, the value for the steric factor could range from one to 
less than $2 / 3.7,8$ Nevertheless, the fact that $P_{\mid-1}$ seoms to be constant as a function of density demonstrates that the steric factor is also a constant of density. An estimate of the scale factor can be found using

$$
\frac{K_{2}}{K_{2}}=\left(\frac{\rho_{1}}{\rho_{2}}\right) \frac{g_{1}\left(R^{*}\right)}{g_{2}\left(R^{*}\right)}
$$

$k$, is the rate for the liquid at density $p_{q}$, where the $i \rightarrow j$ subscript has been dropped, $K_{2}$ is rate at iquid density $\rho_{2}, g_{1}\left(R^{*}\right)$ is the radial distribution function for that liquid density evaluated at some $R^{*}$, and $g_{2}\left(R^{*}\right)$ is the radial distribution function evaluated at $\mathbf{R}^{*}$ for density 2. $\mathbf{R}^{*}$ is the turning point for the most effective collisions and it is assumed that this region is small. In 1971, Davis and Oppenheim derived this equation, using a master equation approach to describe vibrational relaxation in the weak coupling limit in a liquid.9,10 Their theory was thought at the time to apply only to high erequency oscillators, although work by chesnoy seems to indicate otherwise."

This presents the problem of calculating the $g\left(R^{\prime \prime}\right)$ for the oscillator. One approach to this problem was to use the attractive hard spheres pair distribution model by Delalande and Gale. ${ }^{12}$ Thi.: model assumes that the collision rate should be calculated at the hard sphere radius. One then assumes the radial distribution function at $R^{*}$ can be approximated by the carmahan and starling approximation, 13

$$
g\left(R^{\bullet}\right)-g_{H S}(s i g)=\frac{\left(1-\frac{\eta}{2}\right)}{(1-\eta)^{3}} ; \quad \eta=\frac{\pi}{6} \rho \sigma^{3}
$$

where $\sigma$ is the hard sphere contact distance and $\rho$ is the number 
density. The problem with this approximation is that the hard sphere radius which provides the best model for the radial distribution is not necessarily the correct radius at which to evaluate $R^{*}$. A more sophisticated version of this theory was employed by Madden and van Swol." They used wa theory to calculate the cavity distribution function which was then related to the ratio of vibrational relaxation rates in a dilute gas to a dense liquid. This assumed that $g(R)$ could be approximated by a properly chosen hard sphere lluid of the same density. They did not equate $R^{*}$ with the hard sphere diameter used to calculate the radial distribution function. We calculated the radial distribution of an iodine atom in liquid Xe directly. Using the $R^{*}$ calculated from the one dimensional trajectories, 3.7-3.8 $A$, good agreement was found for the scale factors given by equation nine for the different densities.

The scale factors could also have been calculated asing continuum theories. It has been shown that the vibrational relaxation rate is affected by ${ }^{15,16}$

$$
\begin{aligned}
& F(t)=\left\langle\sum_{b} f\left(r_{b}(t)\right) \sum_{c} f\left(I_{c}(0)\right)\right\rangle \\
& F_{b}(t)=\left\langle\sum_{b} f\left(r_{b}(t)\right) f\left(r_{b}(0)\right)\right\rangle
\end{aligned}
$$

Where $F(t)$ is the total force autocorrelation, $F_{b}(t)$ is the binary force autocorrelation and $f(t)$ is the coupling from the liquid to the oscillator at time $t$. Oxtoby has also considered this type of division of the forces. ${ }^{17}$ From these correlation functions and the Golden Rule, the relaxation rate is 


$$
\frac{1}{T_{1}}=\int d t \theta^{\operatorname{tot}} F(t)
$$

Basically, the component of the force autocorrelation spectrum at the oscillator frequency determines the relaxation rate. Figures 2 and 3 show the force autocorrelations and the frequency spectrum for an I atom in liquid $x$ ett $1.8 \mathrm{~g} / \mathrm{cc}$. It has been found that the binary force autocorrelation function frequency spectrum was very similar to the total force autocorrelation function frequency spectrum, down to frequencies of $\approx 50 \mathrm{~cm}^{-1}$. This is evidence for the appropriateness for using IBC theory to model the vibrational relaxation even though $I_{2}$ has such a low vibrational frequency. This implies that the coupling to the bath is weak and sould be treated as linear. Accordingly, stochastic theories should also be appropriate for this system. A stochastic approach should work due to the lineartty of the system and its ablitity to take into account the many body forces through the total force autocorrelation. Smith and Harris have applied a generalized Langevin equation technique to this problem and also found results that agree with the molecular dynamics simulations. ${ }^{18.19}$

Both of the above approaches will fail if the coupling between the bath and oscillator is strong. IBC theory will also fail if the binary force autocorrelation function power spectrum at the appropriate frequency is not the same as the total force autocorrelation. The most probable reason for the two force autocorrelation functions not being the same is if many body effects become more important and provide damping at the oscillators frequency. 
Thus we have shown through experimenta and calculations that IBC theory and continuum theories seem to model well the vibrational relaxation of $I_{2}$ in a simple liquid. This is somewhat surprising given that $I_{2}$ is such a low frequency oscillator and the $I_{2}$ vibrational amplitude is guite large in the upper part of the well. Further experiments in a simple Lennarci-Jones fluid such as Ar are required to test the above theories in a more quantitative fashion.

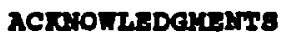

This work was supported in part by a grant by the National Science Foundation and in part by the Office of Naval Research. Molecular Dynamics Simulations were performed at the San Diego Supercomputer center. We also acknowledge the v. S. Department of Energy, office of Basic Energy Sciences, Chemical sciences Division, under Contract No. DE-AC03-76SF00098 for some specialized equipment used in these experiments. 
1. M. F1xoan, J. Chem. Phys., 34, 369, (1961).

2. K. F. Herzteld, J. Chem. Phys., 36, 3305, (1962).

3. M. Chatelet, J. Kieffer, and B. Oksengorn, Chem. Phys., 75, 411, (1983).

4. J. R. Brown, C. B. Harris, and J. C. Tully, J. Chem. Phys., $89,6687,(1988)$.

5. R. L. McKenzie, J. Chem. Phys., 66, 1457, (1975).

6. D. Chandler, J. D. Weeks, and H. C. Anderson, Science 220, 778

7. J. T. Yardley, Introduction to Molecular Energy Transfer, (Academic, New York, 1980).

8. H. K. Shin, Dynamics of volecular collisions part A, Ed. by W. H. Miller (Plenum, New York, 1967).

9. P. K. Dayis and I. Oppenheim, J. Chem. Phys., 57, 505, (1972).

10. P. K. Davis and I. Oppenheim, J. Chem. Phys., 56, 86, (1972).

11. J. Chesnoy and J. J. Weis, J. Chem. Phys., 84, 5378, (1986).

12. C. Delalande and G. M. Gale, J. Chem. Phys., 71, 4531, (1979),

13. N. F. Carnahan and K. E. Starling, J. Chem. Phys., 51, 635, (1969).

14. P. A. Madden and F. van Swol, Chem. Phys., 112, 43, (1987).

15. R. Zwanzig, J. Chem. Phys., 34, 1931, (1961).

16. J. Chesnoy and J. J. Weis, J. Chem. Phys., 84, 5378, (1986).

17. D. พ. Oxtoby, Mol. Phys., 34, 987, (1977).

18. D. E. Smith and C. B. Harris, J. Chem. Phys., 92, 1304, (1990).

19. D. E. Smith and C. B. Harris, J. Chem. Phys., 92, 1312, (1990). 


\section{Figure captions}

Figure 1.

The $I_{2}$ 's average vibrational energy as a function of time in 1.8 $\mathrm{g} / \mathrm{cc} \mathrm{Xe}$ (circles) and in $3.0 \mathrm{~g} / \mathrm{cc} \mathrm{Xe}$ (line) whera the time base of the $3.0 \mathrm{~g} / \mathrm{cc}$ data has been multiplied by 3.0 . The functional form of the relaxation is identical for the two solvent densities. The error bars represent one standard deviation of the average energy for the $1.8 \mathrm{~g} / \mathrm{cc}$ xe solution.

Figure 2.

The total force autocorrelation and the binary force autocorrelation described in equation 11 and 12 are calculated for an I atom in $1.8 \mathrm{~g} / \mathrm{cc} \mathrm{Xe}$. The early time components are very similar.

Figure 3.

The power spectrum of the total and binary force autocorrelation from figure 4. The magnitudes are about the same for vibrational Erequencies above $50 \mathrm{~cm}^{-1}$, which suggests that IBC theory may be appropriate for $I_{2}$ in Xe. 


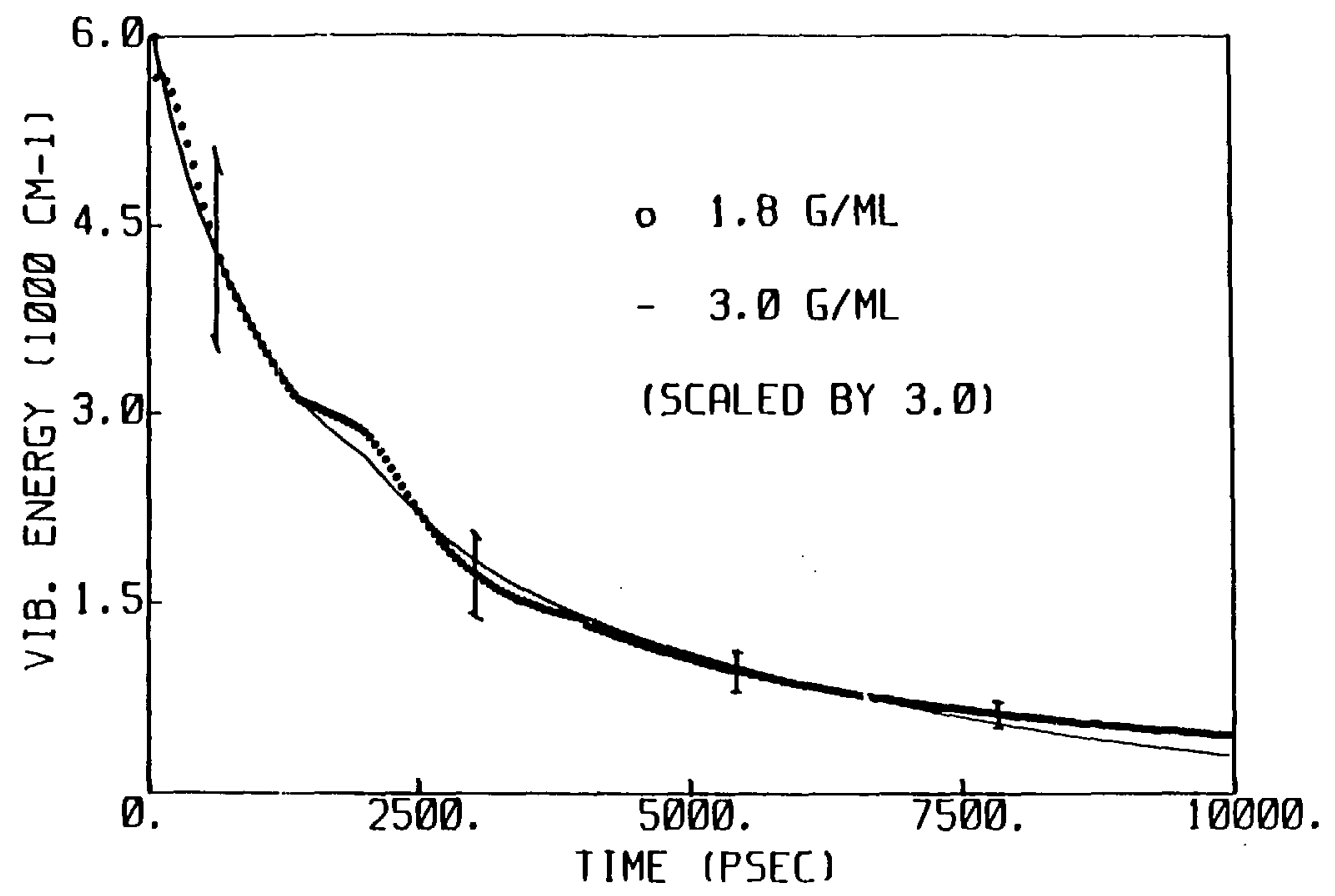

XBL 903-1155 


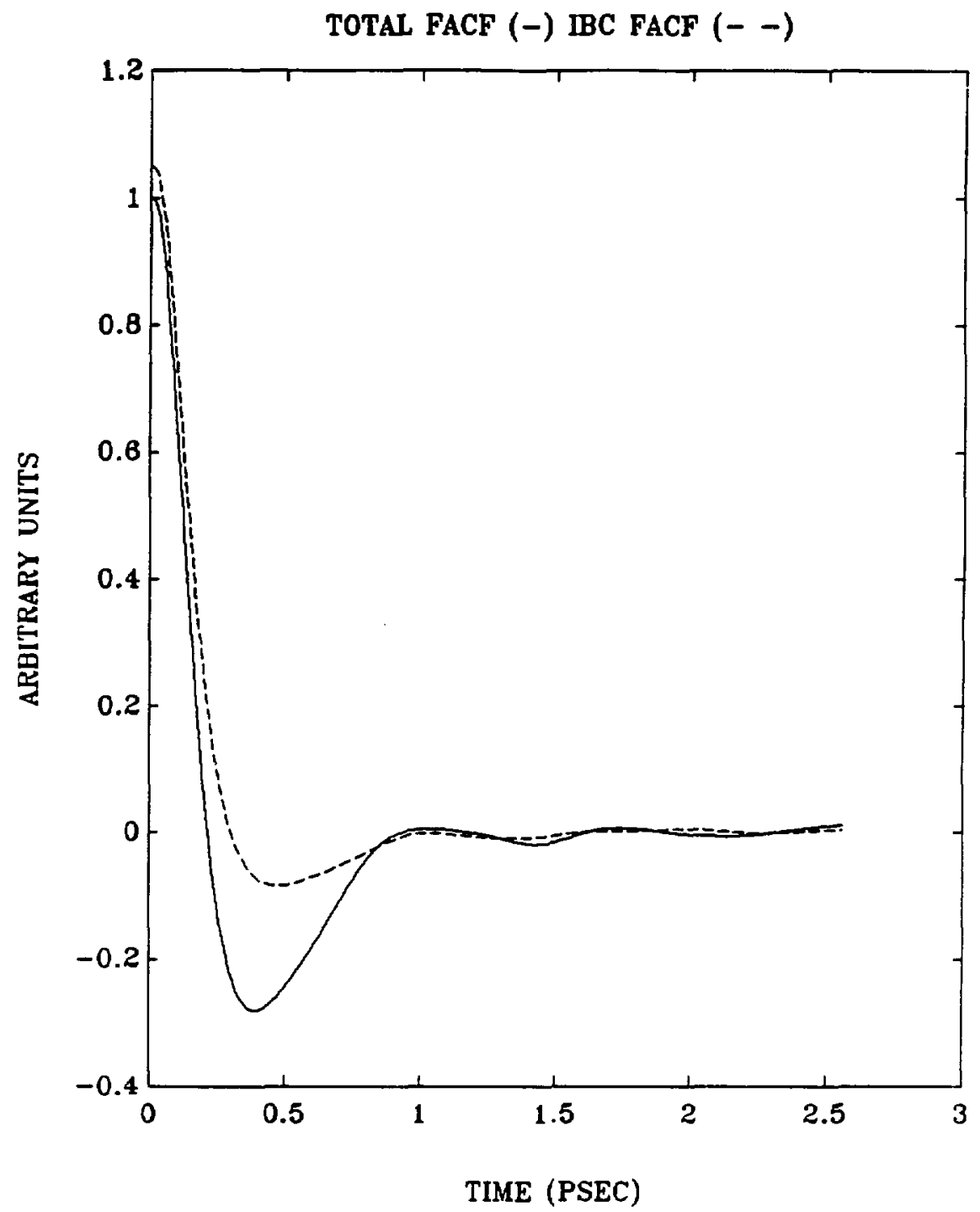




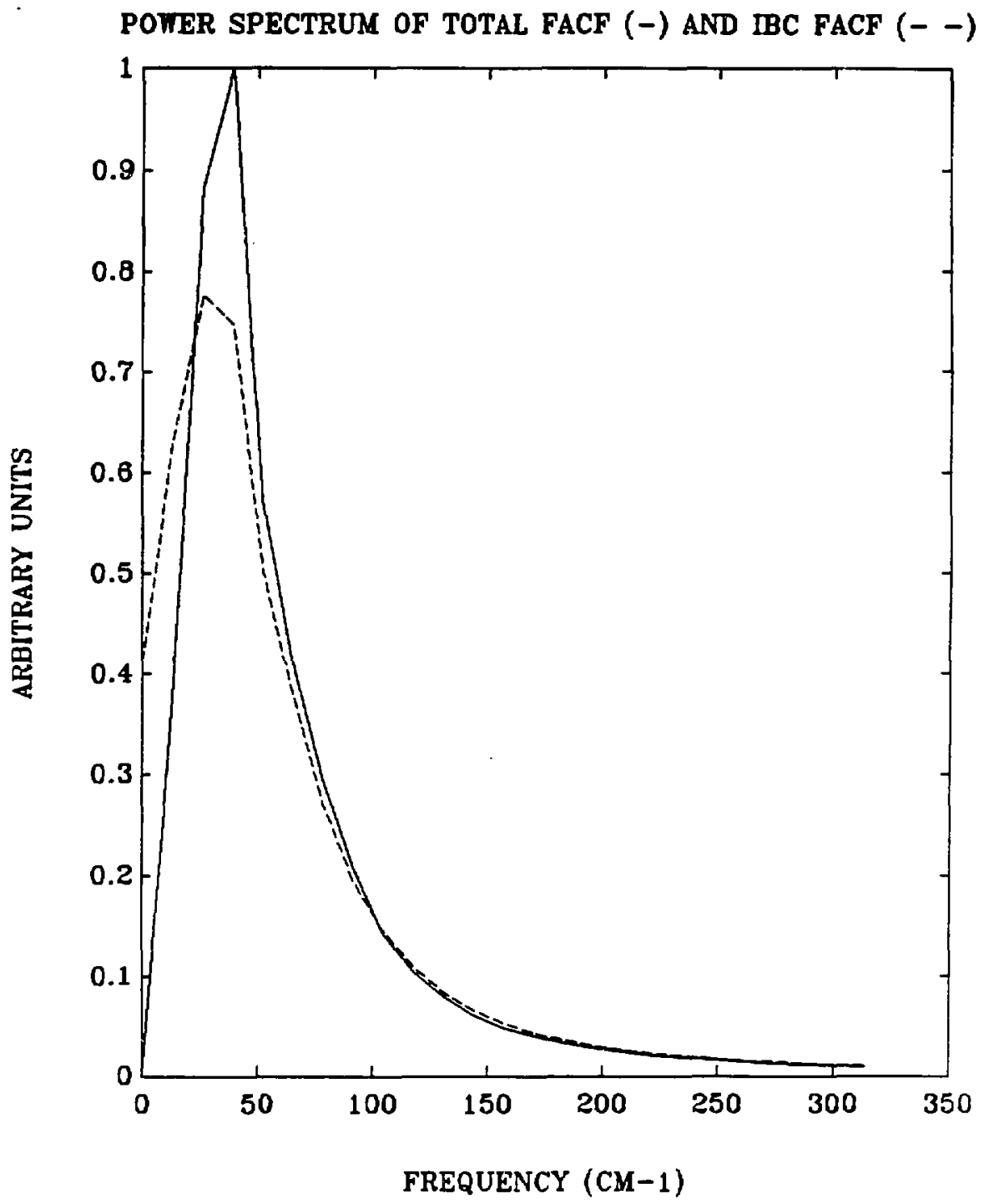

